Insight, part of a Special Feature on A Systems Approach for Sustainable Development in Coastal Zones

\title{
A Systems Approach Framework for Coastal Zones
}

\author{
$\underline{\text { Tom S. Hopkins }}^{1}$, Denis Bailly $^{2}$, and $\underline{\text { Josianne G. Stфttrup }}^{3}$
}

\begin{abstract}
This Special Feature Volume examines the potential value of the Systems Approach Framework (SAF) as a methodological framework for the transition to sustainable development in coastal zones. This article provides insight on the Systems Approach, the theory behind it, and how its practical application to coastal zone systems (CZSs) was developed. The SAF is about information for management through a focus on how to generate a higher, dynamic level of information about complex CZSs and how to render this information more useful to end users through a participatory suite of communication methods. The SAF is an open research methodology that investigates the function of systems in order to simulate specific issues or questions concerning their function. The research articles that are included in this Volume demonstrate examples of coupled multidisciplinary methods integrated into SAF simulations appropriate to a selected policy issue and to the social-environmental conditions of each Study Site Application. Their findings are not the result of funded research projects; instead, they are byproducts of pilot applications conducted to develop and improve the SAF methodology. The final article of this Volume synthesizes these results in the context of the SAF as a higher level instrument for integrated coastal zone management.
\end{abstract}

Key Words: coastal zones; simulation analysis; sustainable development; systems approach

\section{INTRODUCTION}

\section{The SPICOSA Project}

As an Integrated Project (IP), SPICOSA (Science and Policy Integration for Coastal Systems Assessment) developed a product that would stimulate research restructuring and greater integration of new knowledge and methods throughout the European Region. The Project's product is called SAF (Systems Approach Framework). The objective was that it would be a self-evolving, holistic research approach for the integrated assessment of complex systems so that the best available scientific knowledge could be mobilized in support of deliberative and decision-making processes aimed at improving the sustainability of Coastal Zone Systems (CZS). This article provides insight into how theory and practicality were blended to develop the SAF and how it was tested, in order to explain the common basis for the papers in this Volume, which through practical examples demonstrate the value of the SAF.

The Project's integration occurred on several scales, i.e., geographically through testing in the 18 Study Site Applications (SSAs), politically through the participation of 22 countries, institutionally through 54 research institutes, universities, and small enterprises, and scientifically through the multidisciplinary linking of the ecological, economic, social, and governance sectors. The research restructuring was promoted through experimental implementation of the SAF and through professional and academic training. The Project output includes an updateable, web-based support, a users handbook, a model building block library, and SSA models with documentation (http://www.spicosa.eu/). Although the systems approach can be used to construct other frameworks (land use, urban planning, industry, etc.), the SAF is used in this Volume to refer to that developed by SPICOSA for the purpose of managing CZSs. Additionally, the SAF was designed to be an open framework based on systems thinking (Checkland 1981, Capra 1996) that can incorporate aspects of other methodologies or supplement previous approaches to implementing ICZM.

\section{Coastal zones as complex systems}

We use the definition of coastal zone as the long narrow boundary between land and ocean that is a dynamic area of natural and anthropogenic change (cf. LOICZ 1993). This boundary has a prominent subscale resolution due to the local geomorphology and its associated biomes, both of which strongly influence the scale and type of human activities. This makes them more easily identifiable but, in contrast, subjects them to much larger scaled influences. Thereby, most CZSs are strongly open (large mass and energy inputs) and the local natural systems provide only a portion of their total inputs needed to sustain the resident society. The urbanized component imposes an abnormal burden on the surrounding natural systems by generating much greater mass and energy fluxes than their resilience can withstand. CZSs are excellent examples of stressed complex systems by virtue of their numerous interactions and overlapping scales. Studied at local scales, they offer an experimental microcosm for developing methods for improving sustainability in strongly open systems.

CZSs are far from equilibrium and many are changing in a destabilizing direction: i.e., resource consumption is increasing while resource assets are decreasing. This condition underlines the need for the SAF as an instrument for improving our ability to understand, simulate, and communicate changes in CZS. The insertion of the human dimension, including 
culture, heritage, public perception, level of knowledge and communication, is fundamental to a systematic approach to sustainable development (cf. World Commission on Environment and Development 1987).

\section{SYSTEMS APPROACH}

The goal of the Systems Approach is to devise strategies to extract information about the functioning of complex systems that could not have been garnered from a sequence of subsystem-scale studies. It is an issue-oriented investigation and it requires a holistic perspective. This differs from the scientific method, which is more object-oriented, and favors a reductionist focus. Historic use of the scientific method and disciplinary specialization has provided us with an enormous database on the biotic and abiotic composition of systems and much less on their larger scaled interactions. Quantifying issues enmeshed in multiscaled connectivity are a fundamental characteristic of complex systems and the process of quantifying function within this complexity is the fundamental objective of the Systems Approach. It follows then that by adding the Systems Approach to our research repertoire and by incorporating the human dimension into our definitions of systems, we will gain the potential to provide a higher level of information to society for its governance. Undertaking this effort involves confrontation with two principle challenges: that of simulating complex systems and that of creating an effective science-policy interface.

This is not a simple add-on to research, because it requires a broad multidisciplinary training and experience in the skill of "systems thinking", which is a mix of scientific knowledge and intuition needed to understand the characteristics and behavior of complex systems (cf. Capra 1997). A progression of thinkers over the last century has contributed to our understanding of complex systems. The Systems Theory (Bertalanffy 1968) states that complex, nonlinear systems function differently in vivo than a separate scrutiny of their component parts might indicate, which is the basic premise of the Systems Approach. To this we mix in the implication of Godel's theorems that there is not enough information within a system to understand or predict its behavior, i.e., one needs to understand its external inputs, as well as the Heisenberg principle that science is approximate and subject to our method of questioning. More recently, a set of complexity theories has focused on how living systems interact with their environment. Especially important is the concept of self-organization and of living systems possessing characteristics that can be considered as deterministic and reversible only under limited conditions, but we should expect them to also have indeterminate and irreversible characteristics (cf. Kauffman 1995). Self-organization is a function of the diversity and interaction of its components, i.e., many diverse components constructively interacting can evolve to a more complex organization that better optimizes its available resources.
Negentropic reorganization corresponds to the growth part of the Complex Adaptive Cycle (Gunderson and Holling 2002), and entropic disorganization corresponds to the collapse part of this cycle. At the apex in between these opposing directions, systems reach a potential energy maximum, or organizational optimums, relative to the existing environment conditions, but eventually become brittle to changes in environmental disturbances that are more intense or longer than the system's resilience can withstand.

\section{Need for transition methodologies}

Many of the earth's important ecosystems are on the collapse side of this cycle primarily due to anthropogenic events and trends that exceed their resilience. Ecosystem disorganization can be abrupt due to a coincidence of several disturbances, and can cause a "phase shift" in the ecosystem function (e.g., Jamaican coral reefs; Hughs 1994) to higher entropic states as is evident in the decline of marine animals (e.g., Jackson 2001). The combination of disturbances plus continuous trends forces a degradation spiral downward to an irreversible point where a new growth cycle cannot repeat itself because its would-be initial conditions have worsened. Arguably our human societies are presently passing the brittle phase of their Adaptive Cycle where resilience and diversity are decreasing and destabilizing trends and destructive disturbances are increasing, e.g., in fuel, food, climate, and economic debt, due to our overextension of our global niche (cf. Brown 2001). Research is documenting this degradation, and historical evidence (Diamond 2005) suggests that we will follow the majority of previous large societies and collapse due to unwillingness to change when confronted with a diminishing resource base. It is conceptually easy to understand that accepting more people into your hotel than you have beds for is an unsustainable business practice. Yet, this simple analogy applied to the exhaustion of the goods and services provided by natural systems (natural capital) has continued past the sustainable limit since the mid-eighties (Figure 1). This analogy is even worse because our global hotel is not only losing beds (loss of resources) but also more guests are arriving (now beyond the 7 billion mark). It should be likewise easy to understand the relationship between this growing global stress and the responses now manifesting in the social, economic, and environmental sectors.

The fact that the forewarnings of research often go unheeded by policy or society is itself an alarming indicator. Simply documenting the change in natural systems, or providing static indicators of environmental conditions, constitutes an insufficient role for science (Hopkins and Bailly 2012). There are many examples in which indicators and scientific advice have not been successful, e.g., neither the warning of a consistent increase in atmospheric $\mathrm{CO}_{2}$ nor the unheralded peak in fish and food per capita during the 1980s have resulted in less consumption of fossil fuels by those that use it or of 
Fig. 1. The human ecological footprint from 1961 to 2005 (Ewing et al. 2008). The Ecological Footprint is a measure of the demand human activity puts on the biosphere, or Biocapacity (the capacity of an area to provide resources and absorb wastes), in global hectares (ascending red line). In 1986, humanity's consumption began its overshoot with respect to the reference biocapacity of 1961, which has not remained constant but has declined (descending green line), corresponding to its lowered productivity potential relative to its original natural capital. Note that for some commodities the yield (quantity) has remained relatively constant but changed in composition due to technical advances in harvesting that have large unaccounted costs to the environment.

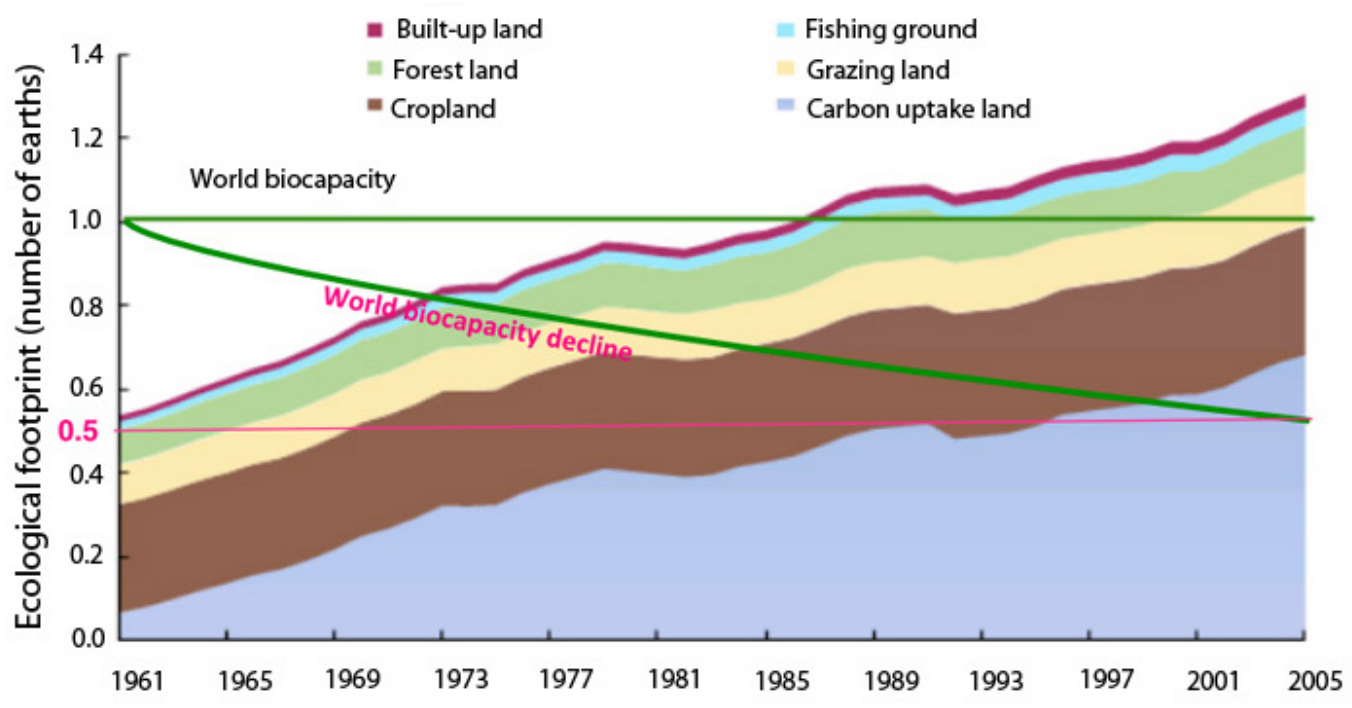

food by those that can afford it. These examples emphasize the argument that research needs to upgrade to dynamic indicators on the strength and number of interactions (Patrizio 2004), to introduce objective simulation analysis into an interactive dialogue useful for policy decision makers and essential for the democratic public.

Research strategies should include a focus on providing precautionary information and prognostic tools to decision makers in order that policy can convert from retroactive regulations to proactive planning and negotiation. Essential to this is the capacity to evaluate the direction of change, as criteria for policy making. It is useless to define goals, if you cannot measure progress toward or away from them. Even if you know the direction policy should take, it requires a platform of cooperation and compromise to make policy effective. Policy for an unfamiliar or future problem is a hard sell, without convincing evidence and significant public awareness. Increasing social awareness and policy effectiveness are social problems. Yet they are ones in which science should have an essential role and that coincide with the goal of sustainable development, which is that of optimizing political and economic strategies for preserving equitable social benefits without damaging the productive potential of the resource base.

\section{SAF CHARACTERISTICS}

\section{A focus on system change}

The SAF must have the capacity to quantify change (past, present, future) in a system and evaluate whether that change is directed toward greater or lesser sustainability. Specifically, we need to know how a studied CZS self-regulates in response to external inputs and through internal interactions. The external inputs from human activities combine, in substance and/or intensity, with those occurring naturally. Under these conditions, ecosystems can degrade quickly, leading inevitably to a spiral of degradation. This is largely because the time scale of degradation is generally quicker than that of recovery, and because many of the human activities develop independently of trends in the state of the natural system. Thus, we must require the best possible information on the function of the ecosystem and on its internal interactions in order to simulate its combined response to projected external influences, including policy choices. Because these are complex systems, we must distinguish in our interpretations between the level of uncertainty introduced due to our methodologies and that due to our lack of knowledge. Finally, to make these responses useful, we must convert them into scenarios adaptable to economic valuations and to social assessments. 
Fig. 2. Coastal Zone System Information Feedback Loops (CZFBL).

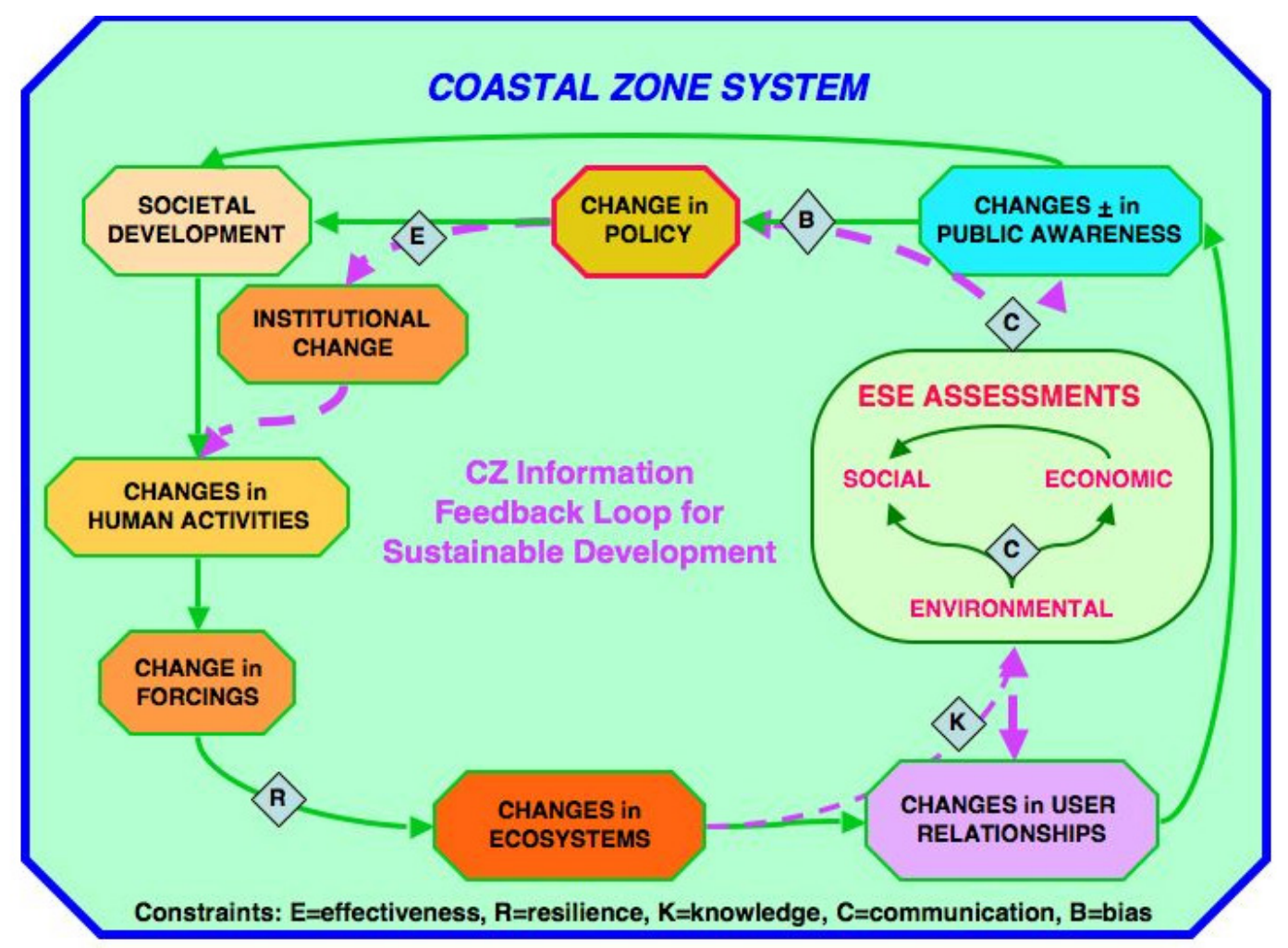

The default loop is shown as the green arrows, and the purple, dashed arrows represent the SAF augmentation of loop. The ESE Assessment box represents the major part of the SAF methodology, which links the assessments of the three ESE dimensions. The default loop is slow in forcing policy to react to problems, many of which become irreversible, while the SAF loop provides quick access to policy makers and the public with objective information on how the CZS responds to changes. When this loop is maintained, it can provide prognostic scenario simulations that allow policy to be precautionary regarding emerging problems, including the efficacy of policy directives. The small diamond boxes represent critical threshold constraints on the interactions between components of the system that need to be properly represented for successful forecasting of policy scenarios.

Parallels between natural systems and social systems are problematic on the basis that humans perceive themselves as different and therefore cannot be quantified in the same manner. Mechanistically they are different, e.g., natural systems operate with available energy as the controlling variable, whereas human systems additionally use stored energy, economic systems use money, social systems use perceptions and cultural paradigms as controlling variables, and humans possess a greater level of consciousness that extends their ability to reorganize. This perception, however, belies the fact that human systems are still subject to the same thermodynamics and most of the ecological principles that govern ecosystems. Furthermore, from a holistic point of view, they are irrevocably connected through their strong codependencies and feedback loops. This discussion has led recent thinkers concerned with global nonsustainability to consider the systems approach as a best option in addressing this challenge, e.g., "Our premise is that the effective pursuit of global sustainability requires a systems approach to the development of polices and intervention strategies" (Fiksel 2006:14).

\section{Coastal zone feedback loop}

The default feedback loop that instigates a corrective policy change in response to an ecosystem degradation is slow, mostly due to the associated economic or social changes that buffer the signal of the loss of goods and services from the natural system (Figure 2). In times of accelerated degradation, the delay can result in long-term irreversibility in the natural system. By supplementing this feedback through the 
Ecological-Social-Economic (ESE) assessments, the SAF increases the potential for the quicker evaluation of changes in the natural system and the identification of remedial policy options. The SAF improves the efficiency and permanence of its loop by requiring an investment of participation of both policy and stakeholders and by contemporaneously disseminating information and knowledge to stakeholders, users, and the public.

\section{Ecological-Social-Economic (ESE) assessment}

In the field of ICZM, the institutional, governance, and cultural dimensions (social) and the economic dimensions are commonly considered as separate dimensions from each other and have been rarely successfully integrated with the ecological dimension in the form of an operational tool at a CSZ scale. Policy decisions generally lack economic evaluations that could provide monetary assessments of the costs and benefits of those social and environmental nonmarket values and consequently tend to be discounted in decisions, which favor short-term, direct market valuation. Receiving little recognition also are the challenges to policy evaluation posed by institutional arrangements, i.e., the design and implementation of property-rights structure or forms of governance. Preferences and social norms also play a significant role that needs to be recognized. The SAF experiment presents initial results toward achieving an improved balance and integration of these social and economic dimensions in relation to the interaction between anthropogenic and natural systems.

The SAF deliberately does not include the dynamics of policy making; it only provides information and a tool for guidance. It is important to delineate the boundary between science and policy as it separates the objective role of science and the democratic role of governance. The SAF attempts to strengthen the science-policy interface by improving both the content of the information and the manner in which it is presented. Thus, the goal of SAF is to provide a higher quality of information, to categorize the information in terms of policy effectiveness in accordance with sustainable development, to include economic assessments of policy scenarios, and to reformat its outputs to the other end user sectors that play a determinant role in policy making.

\section{SYSTEMS APPROACH ADAPTATION}

\section{Characteristics}

The systems approach has had a number of strong proponents interested in understanding complex ecosystems (cf. Jeffers 1978), but its use waned due to a lack of software for representing nonlinear processes inherent in complex systems. This limitation no longer exists. Another fundamental adaptation is the inclusion of the social and economic components and the participation of the client (decision maker). To develop the SAF, the SSA teams were provided with a set of first-guess guidelines that condensed the systems approach into four steps, i.e., Design, Formulation, Appraisal, and Output. Diversity of methodology among the SSAs was encouraged to demonstrate that the SAF is applicable to any CZS and is open to evolving methodologies. The essential characteristic is the use of "systems thinking", which cannot easily be broken down into a rigid set of rules. Necessarily, our development of the SAF prescribed other important characteristics that should be helpful in understanding the case study results of this Volume.

\section{Question driven}

A SAF implementation initiates with the definition of the Policy Issue and a set of Scenarios specific to an observable Impact (dysfunction) in the CZS; and it ends with evaluations of the Policy Issue concerning these issues. The Impact is a recognized, measurable problem in the system, the Issue concerns how and what action might be needed, and the Scenarios are a set of questions to help evaluate which policy options could be pursued. The Simulation Analysis provides the information on this sequence including its delivery to policy and the public. During the SAF development, the Policy Issue was generated through a negotiation between the Study Site Team (Researchers) and volunteers (Stakeholders) from the environmental management sector and representatives from organizations having a vested interest in the local coastal zone. For future applications, a client would propose a policy issue and negotiate with researchers concerning its scope and the resources needed for a SAF application. If the client represents a public office, then Stakeholders from a broad spectrum of interests should be included.

\section{Holistic}

It is holistic in scope in that it must retain cognizance of the entire CZS while it is assessing the internal functionality related to the Policy Issue.

\section{Hierarchical}

The SAF is hierarchical and methodologically flexible in that the simulation can be modified or built upon, and any methodological component can be substituted with another or upgraded. The construction lends itself to the use of ExtendSim ${ }^{\mathrm{TM}}$ model blocks that can be transferred from one application to another.

\section{Iterative}

The SAF is iterative in that its initial focus can be expanded or reduced, dependent on operational constraints and/or new knowledge gained in the process. It has an operational advantage of being rescalable to meet resource or time restrictions to ensure a balance between effort, accuracy, and resolution.

\section{System dependency}

The SAF itself is indifferent to the type of system being analyzed and therefore has value as a common medium for 
the investigation of different types of systems. The complete simulation model for one application is not transportable in its entirety to another. However, many of the model components and the analysis methodologies can be useful to other CZS applications. These adaptations require systems thinking and simulation skills. These same skills provide a capacity to sort out how CZSs respond differently to policy measures, and a capacity to fit policy options to a particular system. Both these capacities are essential to sustainable management.

\section{Information flow}

The SAF places a strong importance on information flow (in addition to mass and energy) through a system, and thus facilitates the inclusion of nonlinear dependencies, threshold switching, controls, and constraints imposed by human society that might be easily represented by other types of analyses.

\section{Communicability}

The results of SAF lend themselves to comprehendible products, i.e., through translation into other formats compatible with the policy dialogue or training. For example, special outputs can include downscaled interactive models that allow an environmental manager or policy to run scenarios and visualize systems' response to changes. Simulation modeling is strongly heuristic, similar to a trial-and-error experiment, and thus has value in both academic and research environments.

\section{Operational tool}

The SAF is far from a onetime decision tool. The methodology is intended to be open and self-evolving, but necessarily grounded in the Systems Approach based on the Systems Theory and recent advances in complex systems. Additionally, it can be cloned and modified for other policy issues, research and management, monitoring system status, revealing knowledge gaps, for resource and economic planning, testing technical options, training, or for monitoring changes in public perception.

\section{Simulation software}

The SAF is a method of approaching a problem and does not necessarily require any specific software. However, in developing the SAF, we chose the ExtendSim ${ }^{\mathrm{TM}}$ software as best satisfying all the characteristics. It can utilize or be coupled to other computational software, such as Matlab, and Excel, etc. Model construction does not require programming in code; rather it is like a Lego set of existing mathematical blocks. In addition, the user can assemble these into a hierarchy of model blocks (e.g., processes, functional components, ESE components, etc.), or the user can program new blocks in a Cbased language. Many of the blocks used by the SSAs are available (http://www.spicosa.eu/). Extend models are formulated at the first derivative in time, which greatly facilitates our focus on change. A constraint, however, is that it can only represent space in a dynamic or virtual sense, e.g., as a box model, an estuary in segments, a river plume based on freshwater content, or a watershed by land use types. A valuable side product of the Project is the development of a coupling of ExtendSim ${ }^{\mathrm{TM}}$ with PCRaster for making calculations on a GIS grid (Vermaat, J. E., S. Broek, B. van Eck, G. Engelen, F. Hellmann, J. de Kok, H., van der Kwast, J. Maes, W. Salomons, and W. van Deursen unpublished manuscript).

Fig. 3. The SAF Virtual System domain with major Components and Interactions.

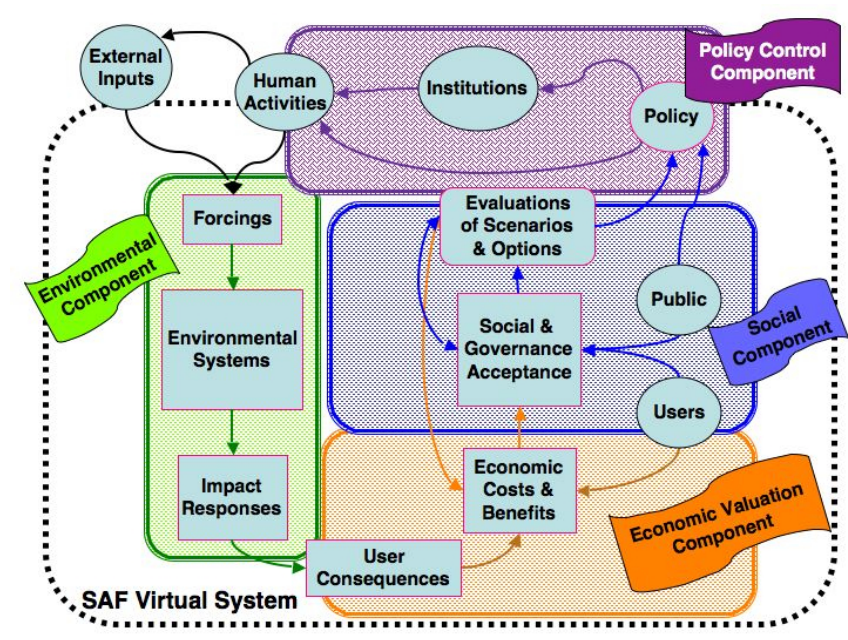

The three Ecological-Social-Economic (ESE) components all have differing dynamics and function, types of information, and spatial-temporal scales. All three need to be simulated as interacting components. The Policy Control is the fourth component; the function of which is not simulated, per se, but the simulated output of the integrated ESE components becomes the information input for Policy. The external inputs to the Virtual System are those inputs from, or external to, the CZS that do not have strong feedback loops with the internal dynamics. However, the inputs that derive from Human Activities internal to the policy domain of the CZS constitute an internal feedback loop through Policy.

\section{Virtual system}

The Virtual System encapsulates the functionality that causes and that responds to the Impact and corresponds to the ESE box in Figure 2. This requires identifying the key components linking the Impact, in the natural system, to causes in the socioeconomic system, and those linking the Impact to Responses in the socioeconomic system (Figure 3). The Virtual System is not extracted from the CZS, but rather it is left connected to it in a graduated manner that gives priority to the more essential connections. The internal interactions are thus identified and sorted according to the level of dependency between components and to the level of resolution required. 
Fig. 4. The locations of the study sites of the SPICOSA Project indicated by a counterclockwise sequence with the names of coastal zones and their primary issues considered. Erratum: In the original published version of Figure 4, study sites $4 \& 5$ were misidentified. The correction was made on 5 April 2012.

\section{SPICOSA STUDY SITE LOCATIONS}

1 Gulf of Riga
2 Gulf of Gdansk
3 Oder Estuary
4 Himmerfjarden
5 Limfjorden
6 Sonderled
7 Clyde Sea
8 Cork Harbour
9 Scheldt Delta
10 Pertuis Charentais
11 Guadiana Estuary
12 Barcelona Coast
13 Thau Lagoon
14 Taranto Mar Piccolo
15 Venice Lagoon
16 Thermaikos Gulf
17 Izmit Bay
18 Varna Bay

1 Gulf of Riga

2 Gulf of Gdansk

3 Oder Estuary

4 Himmerfjarden

7 Clyde Sea

8 Cork Harbour

Scheldt Delta

10 Pertuis Charentais

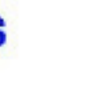

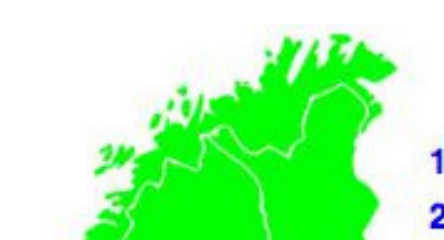

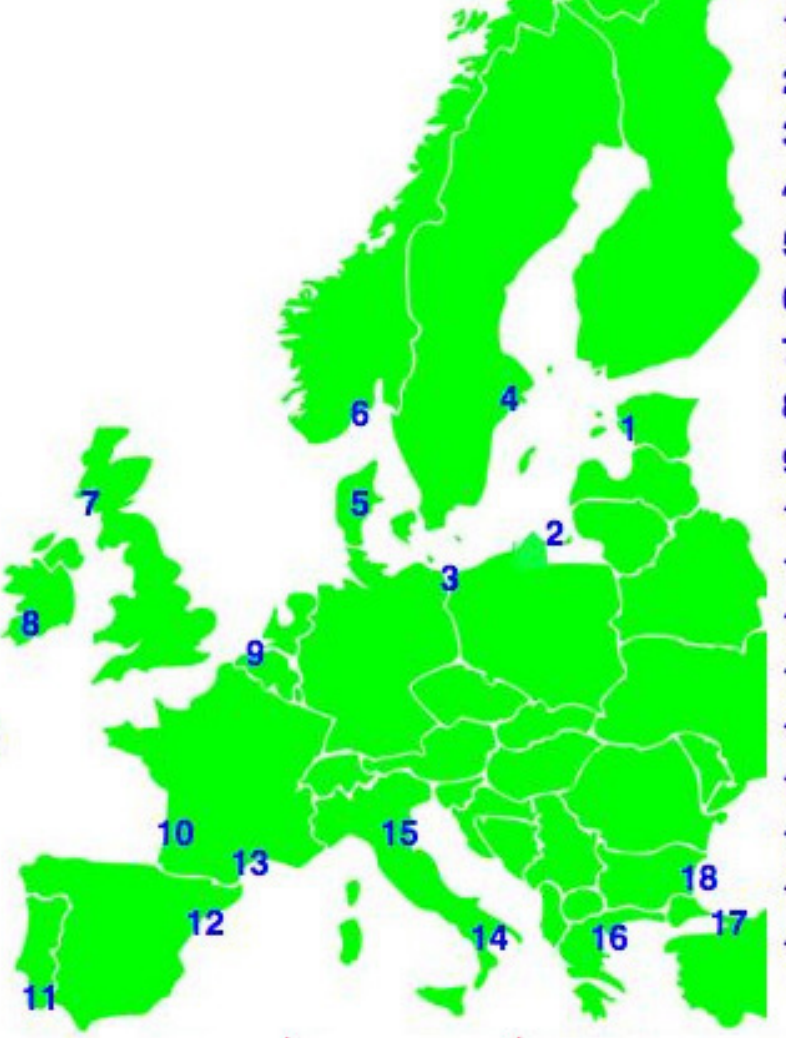

\section{Primary Issues}

\author{
1 Pikeperch \& Fishing
}

2 Water Quality \& Tourism

3 Aquaculture \& $\mathrm{N}$-loading

4 Water Quality \& Sewerage *

5 Mussels \& N-load * Erratum

6 Cod \& Recreation fishing

7 Aquaculture \& Marinas

8 Marinas \& $\mathrm{N}$-loading

9 Agriculture \& $\mathrm{N}$-loading

10 Freshwater \& Agriculture

11 E-coli \& Bathing

12 Discharges \& Beach Quality

13 Aquaculture \& E-coli

14 Mussels \& Waste Discharge

15 Clams \& Fishery

16 Aquaculture \& Illegal Fishing

17 Water Quality \& Real Estate

18 Water Quality \& Tourism
This procedure allows a SAF application to simulate the linkages between human activities and the ESE responses to the Impact in order to guide policy towards sustainable development. Thus, the Virtual System is defined by the components that are directly connected to the identified Impact and those that are indirectly connected to the rest of the System. The hierarchical nature of the SAF allows this boundary to be fluidly defined in a SAF application in a manner such that it can be expanded or contracted depending on changes needed in scope and resolution.

\section{STUDY SITE APPLICATION}

The SPICOSA objective of developing and testing the SAF required that the selected SSAs cover a wide range of CZSs varying in geomorphic type, in environmental conditions, and in the human activities driving these systems. This diversity served another major goal of better understanding the degree to which the natural characteristics of a particular CZS make it more or less sensitive to similar human activities and/or policy controls in other systems. To meet EU-IP objectives, the Project considered necessary a comprehensive distribution of SSAs within the European region (Figure 4) in order to promote consistency in research and policy approaches for European members having different policy stakes and social, economic, or ecological characteristics.

To meet project goals and accommodate the learning process needed, the Project imposed several constraints on the SSAs to ensure coherency of tasks and to optimize information exchange between SSAs: (1) to give priority to the development of the SAF rather than to research aspects, (2) to exchange freely information, methods, and data among the Project, (3) to maintain consistent contact with the client and stakeholder groups, (4) to meet project deadlines. While these constraints differ somewhat from those for a research project, they matched the conditions for a successful SAF application, which must have the capacity to be renegotiated and redimensioned for each application in order that feasibility and resolution are balanced. 
Our intention for the SAF methodology was to jump-start an open, self-evolving procedure for general IZCM use. A successful SAF application depends heavily on systems thinking and, for any specific case, on a combination of the knowledge available and the particularities of a given CZS. To assist future users, the Project has published a web-based Handbook (http://www.coastal-saf.eu/) and a supplementary textbook (Tett et al. 2011). Neither of these advocate a fixed procedure, rather they provide methodological examples and options based on experience gained from the project. For these reasons, we describe here the four basic steps of the SAF application (Figure 5) in the form of brief descriptions of the major tasks for each step.

Fig. 5. Schematic of the SAF implementation for an ICZM Policy Issue.

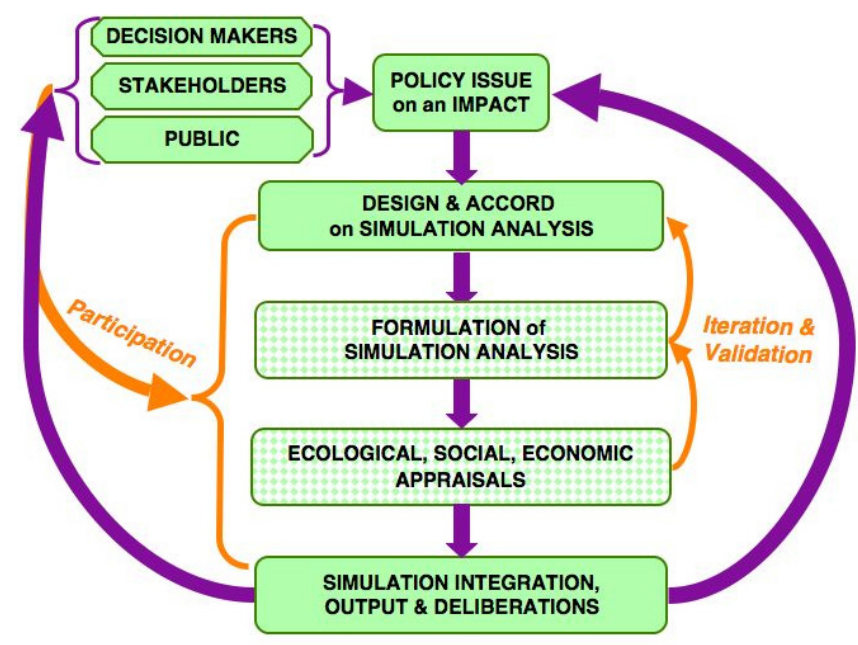

The implementation is a loop that begins with a question (Policy Issue) and ends with the evaluations of the options (Scenarios) concerning that Policy Issue. Essential to the $\mathrm{SAF}$ is the identification of a quantifiable Impact that is the focus of policy attention. A participating stakeholder group follows and interacts with the implementation. The design of the simulation analysis begins with the Impact and its cause in the environmental component and with its response in the social and economic components. The four steps are progressive but allow for iterative corrections up until the final Output Step. The output is formatted for the decision makers, the stakeholders, and for the public. The first and last steps are more holistic, and the second and third steps are more reductionistic.

\section{Design}

The CZS Design defines the objectives (Policy Issue) and gathers the necessary information to initiate the simulation analysis (Virtual System) that addresses the Policy Issue
(Figure 6). The Design Step begins prior to the negotiated agreement with the Client to ensure a mutually understood contract, plans the simulation analysis, and allows for iterations in the planning throughout the Formulation and Appraisal Steps to provide an iterative flexibility to the application.

\section{Policy issue}

From the viewpoint of the Client, the CZS has a Policy Issue and a set of scenarios that need better resolution in order to understand the consequences and effectiveness of associated policy options. From the viewpoint of the SAF Team, an Issue and its Scenarios relate to an observable Impact within the ecosystem and to its Responses within the socioeconomic sectors. These two points of view need to converge through negotiation prior to mutual agreement on the contract for the SAF application.

Fig. 6. Diagram of the activities and products for the SAF Design Step. The activities group into four main tasks related to establishing the Policy Issue, understanding the CZS, constructing the Virtual System, and planning the data and methods required.

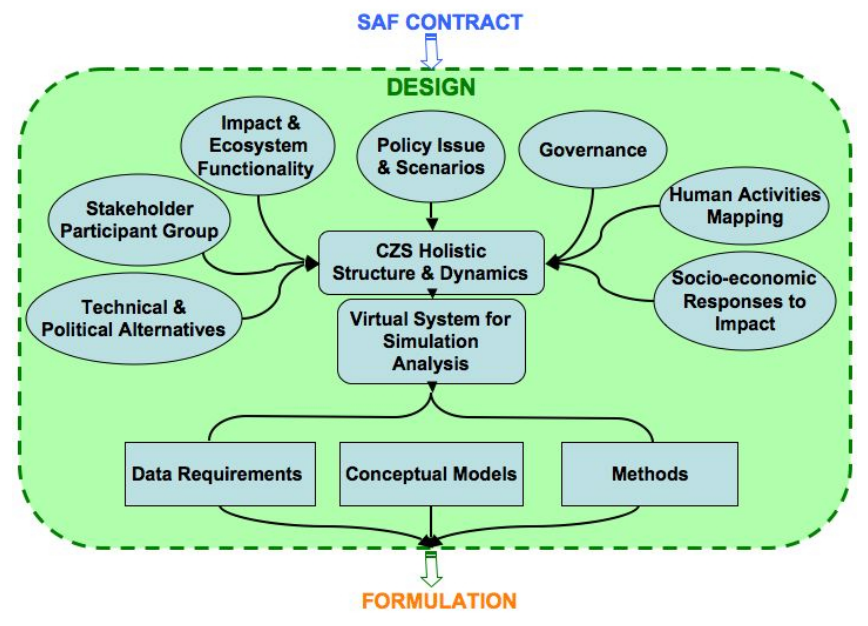

Holistic overview with reductionist focus

The SAF operates on two levels of perspective. The researcher team must maintain a holistic view of the CZS, i.e., potential issues and conflicts confronting policy, interests of the stakeholders, relevant legislation, and information needed for a simulation. Mapping exercises are a good method for obtaining this view, for example, by mapping the human activities, governance, economic activities, and social concerns relative to the Policy Issue, by understanding of the ecosystem's function, and by identifying the goods and services it provides. The Virtual System is then defined as a subsystem of the CZS by sorting the functionalities relevant to the Impact and Response. To do this the researcher must 
take a reductionist perspective in quantifying these functionalities and pay attention to hidden connections to the CZS that might be needed to better simulate unforeseen dynamical shifts in the CZS.

\section{Client and stakeholder involvement}

During the contractual period, the Client and Stakeholders must agree to a participatory role and the SAF Team must agree to maintain an open dialogue throughout the application. This is an essential aspect of the SAF methodology based on the lessons learned during its development, i.e., that participation is necessary to promote an understanding and ownership of the results, that knowledge sharing is essential to cooperation, both of which greatly contribute to the relevance and accuracy of the simulation analysis. The participation will vary depending on the Policy Issue and composition of the Stakeholders. In some cases, Stakeholder engagement may have to be bilateral and separate from the Client.

\section{Conceptual models}

The SAF uses conceptual diagrams to plan the simulation analysis (cf. Odum 1983). The diagram of the CZS is very useful in conveying and discussing the simulation to the Client. Equally important is the subsequent diagram of the Virtual System, which then helps to identify the interactions and variables needed for the simulation analyses and to convey the functionality to the participating Stakeholders.

\section{Data and methods}

These conceptual diagrams also help identify the data needed for the simulation analyses, i.e., data and information, time series inputs, process validation, system hindcasting, industrial and urban wasting, governmental constraints, public perceptions, etc. The methods must be planned and evaluated based on their data requirements and/or limiting approximations required for their use. The final data and methods used should be entered into a documentation report, which is required to validate the analyses conducted.

\section{Formulation}

The Formulation Step represents the functionality of the Virtual System for simulation and interpretation by selecting the most relevant inputs, processes, internal interactions, and constraints and by selecting the most appropriate ones for the simulation (Figure 7). The quantitative part requires that these are formulated into functional model blocks that when connected represent the cause-and-impact chain as well as the impact-and-response chain. The preliminary assemblage constitutes a first-order simulation that can be compared with observations or literature values, checked for stability, and used to identify which second-order information is needed. Individual components can easily be emailed to collaborators. Use of these levels and categories depends on the user's experience and access to validated model blocks.
Fig. 7. Diagram of the activities and products for the Formulation Step. The activities group into several main tasks relating to preparing the data and information, assembling the hierarchical sub-models, conducting validations and sensitivity tests, setting the scope and initiating the qualitative analyses, separately testing the ESE models and determining their linkages, calibrating the simulation model with a hindcast run, documenting data, models and methods.

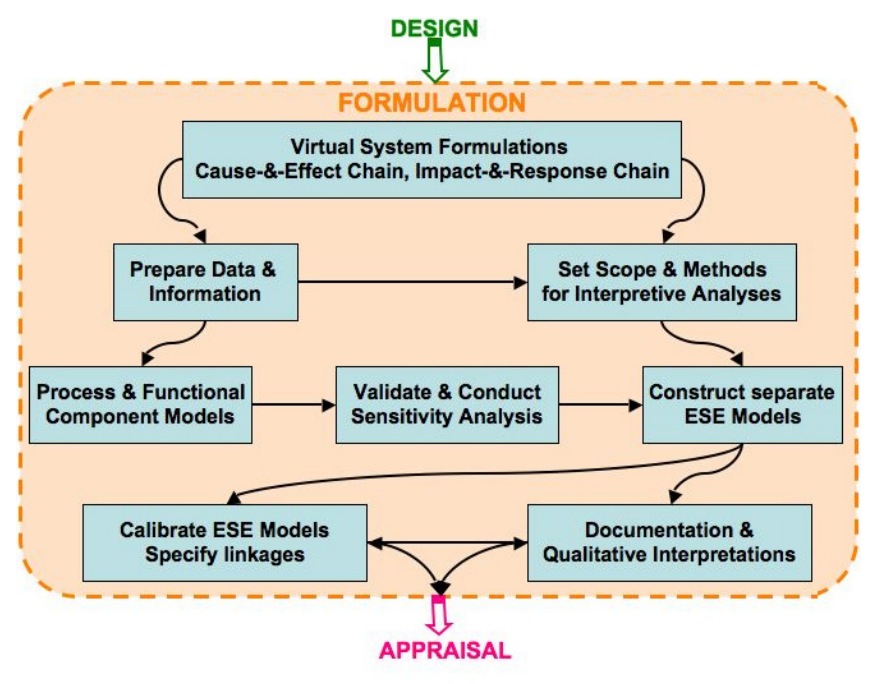

Inputs

The Input information has several levels of availability and of relevance, and the input data has several types, e.g., that for input forcing, process validation, and model hindcasting. Rarely is there a complete and compatible dataset available, which then requires extra effort to find, adapt, or simulate the data as needed. Lack of sufficient data is one of main causes for iteration and/or rescaling during the Formulation. The data source, processing, and values should be reported in the Simulation Documentation at the end of the experiment.

\section{Internal interactions}

We use the term interactions to include processes, input functions, feedback loops, etc. Inputs of mass, energy, and information commonly are converted on entry into the system, e.g., light to photosynthesis, fresh water to potential energy, public perception to resource use, etc. These are essential to the simulation in terms of separating the variability in the system to natural and anthropogenic causes. The ability of the ecosystem to optimize the use of these inputs is dependent on a set of internal interactions that redistribute and recycle mass and that conserve the energy characteristic of complex systems. In a first-order simulation, only the main trajectories of these inputs are tracked through the cause-and-effect chain and the effect-and-response chain. If the output of the first- 
order simulation has an acceptable accuracy, then the sensitivity of the output is used for determining which of the formulations generate the least error. Approximations are acceptable only if they do not reduce the credibility of the model (error must be documented), which can occur by oversimplifying the dependent variables of key processes, for example, the use of empirical constants in place of dynamic variables isolates a process from its connections to the rest of the system. Any simplifications made by eliminating secondary processes must be considered carefully, in order to retain enough connectivity within the system to anticipate the errors associated with overlinearizing or limiting our representations.

\section{Hierarchical Structure}

ExtendSim ${ }^{\mathrm{TM}}$ utilizes a hierarchical structure of mathematical blocks to represent calculations of, e.g., a process, a cluster of processes, or a subsystem. Thus, a complete system model consists of a suite of interconnected blocks of varying complexity. This characteristic renders a model amenable for a SAF application for three main reasons: the holistic perspective can be preserved, any block can be "unplugged", upgraded, validated, and reinserted, and iterations on the model's scope or objectives can be accommodated.

\section{Interpretive analyses}

Complementary interpretive analyses are conducted to describe the model construction, its results, and to accommodate portions of required simulation analyses that cannot be directly modeled. The type of interpretive analysis ranges from input data analysis, to the interpretation of subjective questionnaires, to the presentation of simulation results. The utility of any model, and particularly a multidisciplinary simulation, rests on the quality of science used to interpret the results. The methods chosen for these analyses are user, resource, and system dependent. Much of the skill of simulation modeling rests in the choice of methods and approximations used.

\section{Documentation}

Data source, description of the data (methods, frequency, units), and data handling should be reported throughout the process including methods used for upscaling or downscaling, approximations, or conversions. Stakeholder meetings, opinions, and contributions also need to be documented.

\section{Appraisal}

The Appraisal Step assembles the integrated Simulation Model through final linkages between the ESE component models (Figure 8). This model is suitable for evaluating the scenarios and for completing the accompanying Interpretive Analyses. The separation between the Appraisal and the Formulation Steps is arbitrarily set at the point where the quantitative focus shifts from preparation to results. The ESE component models need to be run and interpreted separately for sensitivity and error analyses. It is also necessary when there is a temporal incoherence in the calibration data needed for each ESE component. Coordinated tests of the linkages are needed to check the mutual interactions between ESE components. This is particularly important for feedback loops when the time scale of reversibility differs, e.g., the time scale of recovering a seafood product may greatly exceed the tolerable unemployment time of the fishermen. The ESE component models are then coupled to construct the simulation hindcast model, which is run over a sufficiently long time period to confirm the model stability, response to a policy change, and accuracy of results calibrated against available observational data.

Fig. 8. Diagram of the activities and products for the Appraisal Step. The primary activities are to make sure that the ESE models are calibrated with observations, conduct the associated analyses, assemble the final simulation, run the scenarios, interpret all the results, and format them as research products.

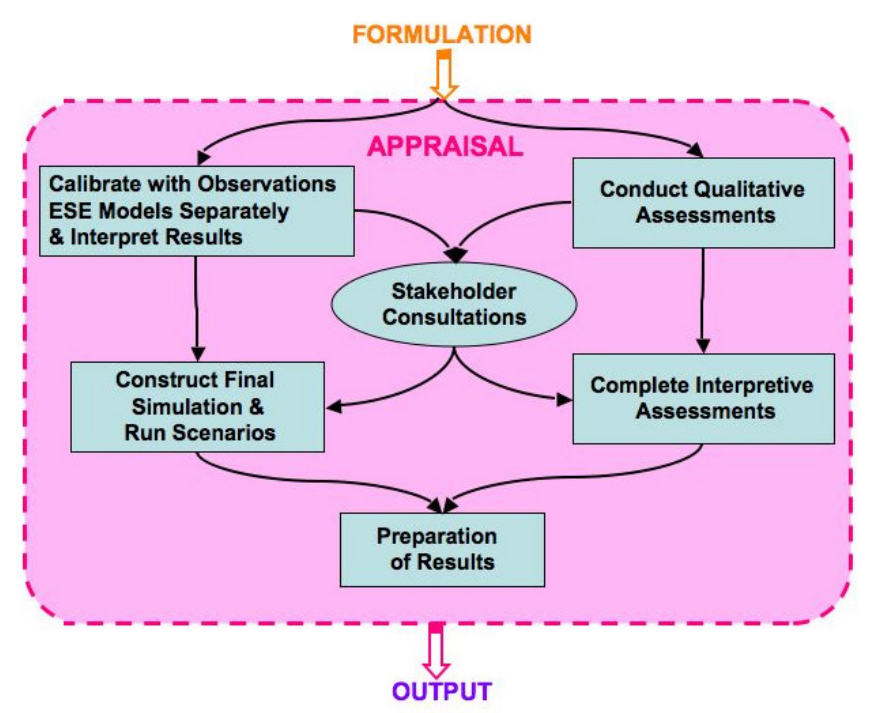

For the qualitative analyses, the initiation of the assessments and their completion is a continuous iteration process from Formulation through Appraisal. An iterative overlap between Appraisal and Output is maintained regarding the expected format and specifications of the Output products. The interpretation of results should succeed in addressing the system complexity (e.g., feedback loops, thresholds, potential phase shifts, sensitivity, etc.) revealed in the simulation and needed for the communication with decision makers (Engelen et al. 2003). During the final phase of the Appraisal, participatory sessions should be conducted with the Client and Stakeholders to better define the expected Output products.

\section{Output}

Again, the separation between the Appraisal and Output is arbitrarily set at the point that the simulation analysis is completed. The purpose of the Output is to integrate and 
organize the qualitative and quantitative information resulting from the SAF for written and interactive presentations (Figure 9). The contents vary between interpretations (qualitative descriptions, dynamic indicators, error and effectiveness critique, recommendations), ESE analyses of scenarios, and interactive deliberations conducted with the Client, with Stakeholders, and with the public. The recipient focus groups vary, e.g., science-policy consultations, stakeholder forums, media releases, and academic material. A multifaceted approach is used to improve the transfer of knowledge. Presentations use a well-structured set of techniques through multimedia, shared podium, moderators for interactive discussions, and concise hardcopy summaries. These efforts are done in the context of investing in greater cooperation between local researchers and environmental managers and between stakeholders common to an issue, and in greater public awareness.

Fig. 9. Diagram of the activities and products for the Output Step. The main activities are to translate and integrate the final results, consult with the Client/Stakeholder group, construct presentations for various users (media, academic, training, online interactive models, etc.), and submit a summary report as specified in the contract with the Client.

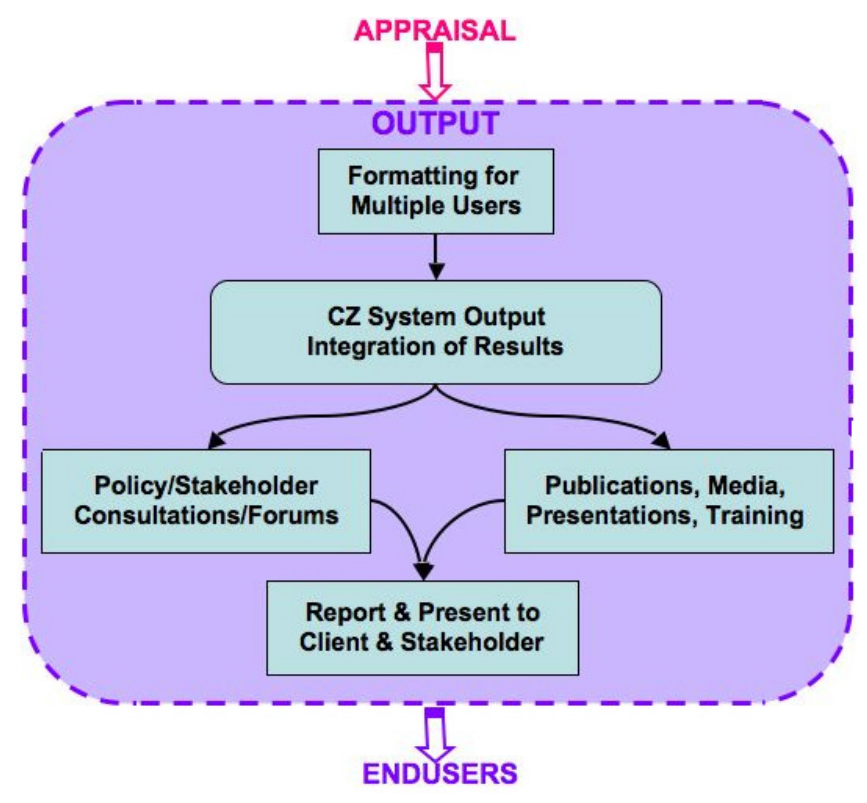

\section{DISCUSSION}

A practical requirement of the SAF was that it could be implemented on a policy time scale of roughly a year. The development took the four-year duration of the Project. Much of this time was expended with the learning curve, data acquisition, personnel problems, and funding delays. The development had a wide scope with two priorities: testing the implementation and establishing support activities. The
Project had a node of work tasks devoted to writing the guidelines for the execution of the four SAF steps. This initial procedure was then modified through feedback from all the SSAs, which was used to write a web-based handbook for future users. Another node focused on support activities, e.g., model support, library of model blocks, data portal, alternative strategies, and external communications, the products of which are all available electronically (http://www.spicosa. $\mathrm{eu} /$ ). We also invested in academic activities to create courses and curriculum in systems and training activities for professional training in SAF for ICZM. All of this support investment was designed to shorten the implementation time and establish a growing community of users.

Whether the exposure to the SAF was sufficient to generate its continued use and evolution remains to be seen. On the one hand, the learning curve is experiential and made difficult by a lack of training in systems science. Initially, only a few researchers had worked with the systems approach and/or had closely collaborated with ESE researchers other than from their own discipline. Most SSA partners had to recruit postdocs to supplement their SAF Team, especially in the social and economic disciplines. All SSAs had difficulty finding sufficient existing data, and funding constraints prohibited any field observations. During the Design Step, it became very clear to many SSA Teams that the work was truly multidisciplinary. Apart from language problems in translating English terms to local languages for stakeholder engagement, the teams had to deal with generic words, which had different meanings in the different disciplines. The priority goal of the Project was to develop the SAF methodology, and not to specifically do research. Despite these limitations, all the SSA exercises have contributed a rich and diverse set of applications to the SAF development.

As a by-product of their applications, the SSAs demonstrated the research value of the SAF through clearer identification of policy options, a better understanding of how a CZS functions, and the longer term benefits of sustainable development. For example, policy makers, stakeholders, and researchers alike enthusiastically accepted the possibility of a tool for quantifying policy options and to unite common interests for sustainable benefits. Some SSAs found it very useful to discuss the sub-models with clients and stakeholders during the formulation step. The stakeholders either confirmed that the results reflected their understanding of the system or they critiqued the results. In some cases, they came with suggestions to alter the models either by providing a better understanding of processes or helping to improve databases by providing new data not previously available. In such cases, the new data greatly improved the models and it was possible to ensure anonymity during presentation of results.

The articles in this Volume discuss examples in the two focus areas of the SAF: simulation analysis and the science-policy interface. We ask the readers not to consider these articles as results of individual research projects, but as a set of 
multidisciplinary research themes and methods that demonstrate the potential value of the SAF. The final article in the Volume discusses further the need and value of the SAF and provides a comparative analysis of the ensemble results (Hopkins, T. S., D. Bailly, J. G. Støttrup, A. Sandberg, R. Elmgren, and G. Glegg, unpublished manuscript).

Responses to this article can be read online at: http://www.ecologyandsociety.org/voll6/iss4/art25/ responses/

\section{Acknowledgments:}

The authors wish to acknowledge the EU Environment FP6 for its foresight and funding in the form offarsighted objectives to stimulate research towards the needs of the evolving EU community. Most of all we thank the several hundred researchers who have contributed, persevered, and brought an optimistic enthusiasm to the completion of Project SPICOSA. We wish to recognize the Partners' cooperation and willingness to support the Project, and we especially thank Maurice Heral and Daniel Roy of IFREMER for their expert and conscientious attention to the administration of the Project.

\section{LITERATURE CITED}

Bailly, D., and T. S. Hopkins. 2007. Science and policy integration for coastal systems assessment (SPICOSA). EU Integrated Project, Sixth Framework Program, Global Change and Ecosystems. [online] URL: http://www.spicosa.eu/

Bertalanffy, L. von. 1968. General systems theory. George Braziller, New York, USA.

Brown, L. 2001. Eco-economy. W.W. Norton and Company, New York, USA.

Capra, F. 1997. The web of life. Anchor Books, New York, USA.

Checkland, P. 1981. Systems thinking, systems practice. Wiley, Chichester, UK.

Diamond, J. 2005. Collapse: how societies choose to fail or survive. Viking Books, New York, USA, and London, UK.

Engelen, G., I. Uljeer, K. L. Van de Ven. 2003. WADBOS: integrating knowledge to support policy-making in the Dutch Wadden Sea. Pages 513-537 in S. Geertman, and J. Stillwell, editors. Planning support systems in practice. Advances in spatial science. Springer-Verlag, Berlin, Germany.

Ewing, B., S. Goldfinger, M. Wackernagel, M. Stechbart, S. M. Rizk, A. Reed, and J. Kitzes. 2008. The ecological footprint atlas 2008. Global Footprint Network, Oakland, USA.
Fiksel, J. 2006. Sustainability and resilience: toward a systems approach. Sustainability: Science, Practice, \& Policy 2 (2):14-21. [online] URL: http://sspp.proquest.com/archives/v ol2iss2/0608-028.fiksel.html

Gunderson, L. H., and C.S. Holling, editors. 2002. Panarchy: understanding transformations in human and natural systems. Island Press, Washington, D. C., USA.

Hopkins, T. S., and D. Bailly. 2012. The role of science in the transition to sustainability: the systems approach framework for integrated coastal zone management. In E. Moksness, E. Dahl, and J. Støttrup, editors. Integrated coastal zone management. Second edition. Wiley-Blackwell. In press.

Hughs, T. 1994. Catastrophes, phase shifts, and large-scale degradation of a Caribbean Coral Reef. Science 265 (5178):1547-1551. http://dx.doi.org/10.1126/science.265.517 $\underline{8.1547}$

Jackson, J. B. 2001. What was natural in the coastal oceans? Proceedings of the National Academy of Science 98 (10):5411-5418. http://dx.doi.org/10.1073/pnas.091092898

Jeffers, J. N. R.. 1978. Introduction to system analysis: with ecological applications. E. Arnold, London, UK.

Kauffman, S. A. 1995. At home in the universe: the search for laws of self-organization and complexity. Oxford University Press, Oxford, UK.

LOICZ. 1993. Land-ocean interactions in the coastal zone. [online] URL: http://www.loicz.org/index.html.en

Odum, H. T. 1983. Systems ecology: an introduction. WileyInterscience, New York, USA.

Patricio, J., R. Ulanowicz, M. A. Pardal, and J. C. Marques. 2004. Ascendency as an ecological indicator: a case study of estuarine pulse eutrophication. Estuarine, Coastal and Shelf Science 60(1):23-35. http://dx.doi.org/10.1016/j.ecss.2003.11.017

Tett, P., A. Sandberg, and A. Mette, editors. 2011. Sustaining coastal zone systems. Dunedin Academic Press, Edinburgh, Scotland.

World Commission on Environment and Development. 1987. Our common future (the Brundtland Report). Oxford University Press, Oxford, UK. 